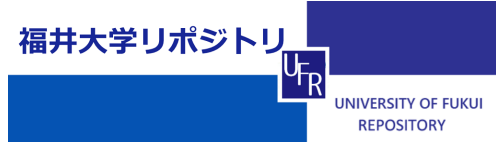

Convective I nstabi I ity of the Bernstei n Wave Propagat ing Obl i quel y to the Nagnet i c Fi eld in Beam Pl asma Syst em

\begin{tabular}{|l|l|}
\hline $\begin{array}{l}\text { j our nal or } \\
\text { publ i cat i on t i t l e }\end{array}$ & 福井大学工学部研究報告 \\
\hline vol une & 22 \\
\hline number & 2 \\
\hline page $r$ ange & $273-286$ \\
\hline year & 1974 -09 \\
\hline URL & ht t p: //hdl . handl e. net /10098/4666 \\
\hline
\end{tabular}


MEMOIRS OF THE FACULTY OF ENGINEERING

FUKUI UNIVERSITY

VOL. 22 No. 21974

\title{
Convective Instability of the Bernstein Wave Propagating Obliquely to the Magnetic Field in Beam-Plasma System*
}

\author{
Toshitaka Idehara, ${ }^{* *}$ Mitsuyoshi TakedA, ${ }^{* *}$ Yoshio IshidA**
}

(Received Apr. 15, 1974)

\begin{abstract}
The Bernstein wave propagating obliquely to the magnetic field is excited by the coaxial probe and is heavily damped by the Landau and/or cyclotron damping with respect to the direction along the field, whose propagation direction may be coincident with that of the maximum value of group velocity $(\partial \omega / \partial \mathbf{k})$. When a rather weak electron beam is injected, the wave is amplified due to the convective instability, whose wave vector $\mathbf{k}$ is determined as follows: the wave number component $k_{\|}$paralell to the field satisfies the Cherenkov excitation condition $\left(k_{\|} \mathbf{v}_{b} \approx \omega\right)$, and then, the component $\mathbf{k}_{\perp}$. perpendicular to the field is determined by the dispersion relation $K\left(\omega, k_{\|}, k_{-}\right)=0$. Increasing the intensity of the electron beam above the threshold value, the spontaneous excitation of the wave is observed, which can be explained consistently as the result of the convective instability of thermal noise in plasma.
\end{abstract}

\section{Introduction}

In a warm magnetized plasma, the Bernstein wave has been investigated as one of the interesting waves, because it is the most generalized wave in a high frequency region $\left(\omega \gtrsim \omega_{c}, \omega_{p}\right.$; where $\omega_{c}$ and $\omega_{p}$ are the electron cyclotron and plasma frequencies, respectively). Moreover, from the viewpoint of the wave phenomenon, it has the unique characteristics that it appears as a forward and/or a backward wave with varying the plasma parameters $\left(\omega_{c}, \omega_{p}\right.$ and electron temperature $T_{e}$ ). The wave is predicted theoretically by Bernstein ${ }^{11}$ and its dispersion relation is fully analized by Crawford et $2 ._{.}^{2)}$ in the cases of plasmas with the various velocity distribution function of electrons.

The experimental investigations of the wave are divided into two parts; one of them is the propagation experiment, ${ }^{3)}$ which is concerned with only the wave prapagating perpendicularly to the magnetic field, because the wave has not resonant particles and can propagate without Landau or cyclotron damping. The experiment

*Reported in part in Phys. Letters 46A (1974) 409. **Department of Applied Physics. 
confirms that the observed dispersion relation is coincident with the theoretical expectation. ${ }^{2)}$ While, the propagation of the wave having the propagation component along the field (hereafter, denoted by 'oblique propagation') has been scarcely investigated experimentally, because of heavy damping of the wave. The other is the experiment of instability of the wave in an electron beam-plasma system," which is concerned with the oblique propagation satisfying the synchronization condition ( $\omega-$ $\left.\mathbf{k}_{\|} \mathbf{v}_{b \|}=\mathrm{n} \omega_{c}\right)$. Therefore, the instability experiment has not been supported by the oblique propagation experiment.

In this paper, the characteristics of the oblique propagation of the wave are investigated experimentally, and compared with the calculation of the dispersion relation. Then, when the electron beam is injected, the instability of the wave occurs, because the growth rate of the wave exceeds the damping rate of the wave. These excitation of the wave can be explained by the convective instability.

In the next section, the experimental apparatus and experimental procedures are explained, in \$3, the experimental results and discussions are expressed and in final section the conclusion of the paper are described.

\section{Experimental Apparatus and Procedures}

In order to investigate the propagation of waves and the instability of the wave due to the interaction of an electron beam with a plasma, it is desired that a Maxwellian plasma is produced and an electron beam is injected into this plasma, both parameters of plasma and beam being varied independently. Considering such a requirement, we have set up the apparatus which is consisted of three regions, that is, the dc discharge region, the plasma diffused region (or the beam-plasma system) and the beam generated region, as shown in Fig. 1. Argon gas of the pressure of about $1 \sim 2 \times 10^{-2}$ torr is fed into the discharge region and, by using a method of differential pumping, the gas pressures of the plasma diffused and the beam generated regions are maintained at about $3 \sim 7 \times 10^{-4}$ and $0.8 \sim 1 \times$ $10^{-4}$ torr, respectively. An external magnetic field is applied along the tube axis and its intensity distribution on axis is shown in Fig. 1. The plasma is produced by a dc discharge and diffused through an orifice $(10 \mathrm{~mm}$ in diameter and 200 $\mathrm{mm}$ in length) and a hole ( $8 \mathrm{~mm}$ in diame-

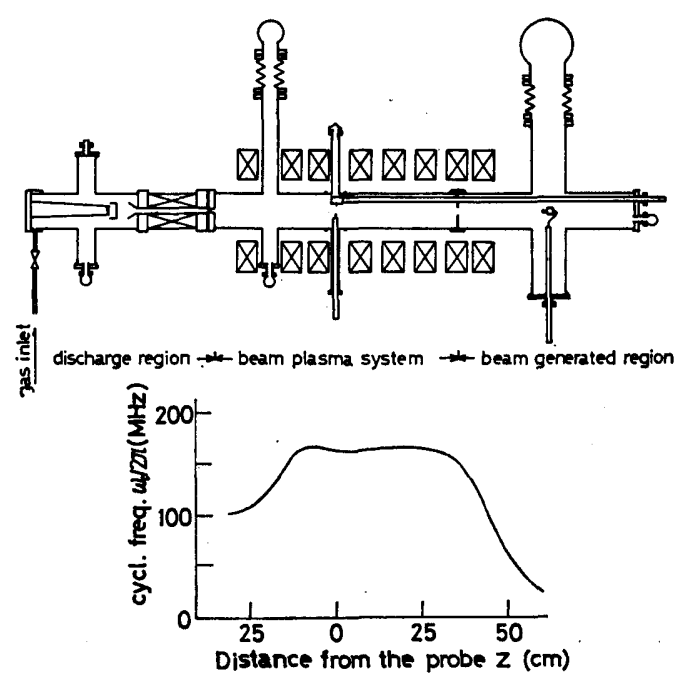

Fig. 1 Experimental apparatus and the distribution of the magnetic field intensity. 
ter) at the center of an anode into the plasma diffused region along the external magnetic field. In the region, the field is uniform within 3 percent and its strength is 60 gauss, which is cor responding to $\omega_{c} / 2 \pi=168 \mathrm{MHz}$. The plasma is supported by the field near the axis of glass tube ( $95 \mathrm{~mm}$ in diameter and $700 \mathrm{~mm}$ in length). The plasma density profile in the radial direction is shown in Fig. 3 . When the discharge current $I_{d}$ is varied from 2 to $23 \mathrm{~mA}$, the plasma density $\mathrm{n}_{p}$ is varied from $8 \times 10^{8}$ to $9 \times 10^{9} \mathrm{~cm}^{-3}$ but the electron temperature is constant at about 6 $\sim 10 \mathrm{eV}$ in the region. The principle of the apparatus is similar to the TP-D machine at the Institute of Plasma Physics, Nagoya University. ${ }^{5}$

An electron beam is produced by the Pierce gun in the beam generated region, and is injected into the plasma diffused region through a hole of $15 \mathrm{~mm}$ in diameter. When the accerelation voltage $V_{b}$ of the beam is changed from 50 to $500 \mathrm{~V}$, the current of the beam $I_{b}$ changes from 0.18 to $3.1 \mathrm{~mA}$. (The perveance of the gun being about $5 \times 10^{-7} \mathrm{AV}^{-3 / 2}$.) The electron density of the beam $n_{b}$ is varied from $1.5 \times 10^{8}$ to $4.5 \times 10^{8} \mathrm{~cm}^{-3}$, but the temperature $T_{b}$ of the beam is constant at about $0.3 \mathrm{eV}$.

In order to excite and receive the wave, three coaxial probes are inserted in the plasma diffused region, one of them being movable radially and the other two being movable axially. The signal of the wave excited by a probe is detected using another probe and its propagation pattern is measured and recorded by the interferometer system as shown in Fig. 2. The delay line is used in order to determine the direction of the wave propagation. When an intense electron beam is injected and the wave is excited spontaneously,

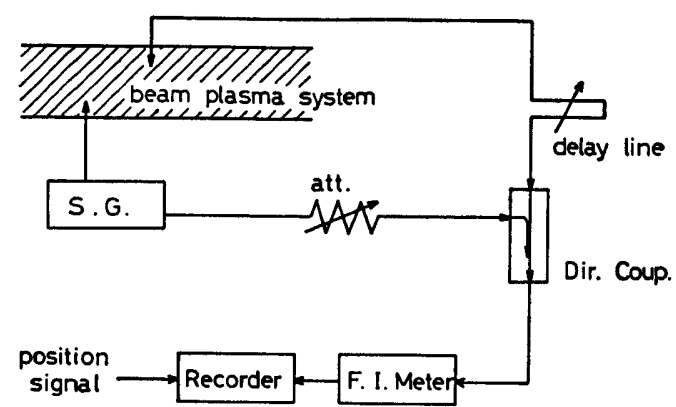

Fig. 2 The interferometer system.

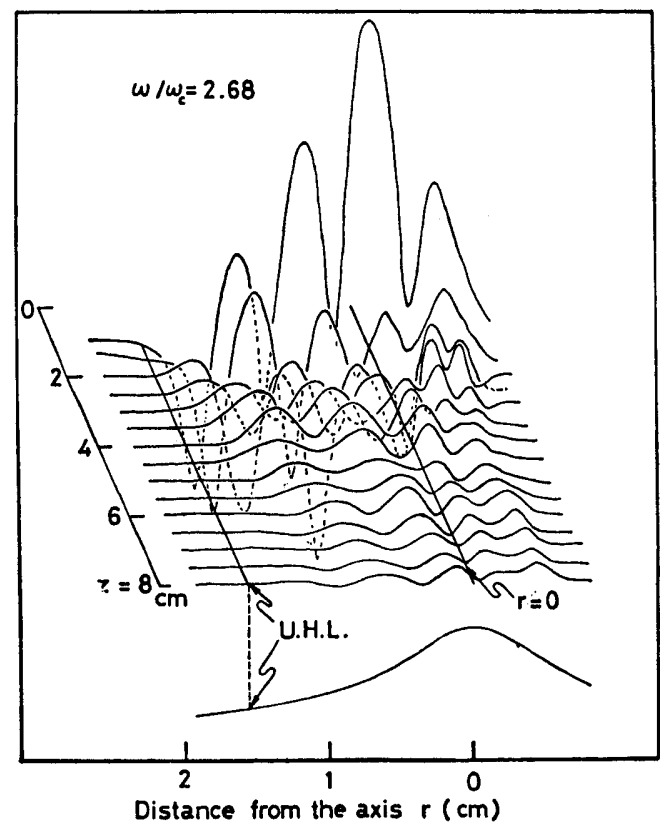

Fig. 3 The propagating wave patterns excited by a coaxial probe and the radial density profile of plasma. The exciting probe is situated at $r=z=0$. UHL shows the upper hybrid layer. $\left(\mathrm{n}_{p}\right)_{\max }=5.6 \times 10^{9} \mathrm{~cm}^{-8}$ $\left(\omega^{2} p / \omega^{2} c=16.0\right)$.

the self correlation is measured by using two of them. From the recorded wave patterns, the wave number and damping rate (or growth rate) are determined. The 
excited and/or received frequency is varied from $168 \mathrm{MHz}$ to $500 \mathrm{MHz}$. When the intensity of received signal is determined, it is compared with and equalized to that of the impulse generator by inserting a known value of attenuation in the transmission line from the plasma to the field intensity meter.

\section{Experimental Results and Discussions}

\subsection{Observation of the wave propagating obliquely to the field}

At first, the propagation characteristics of the wave excited by a probe are measured and compared with the calculated dispersion relation. The wave is excited by using one of the $z$-probes situated on the tube axis (i. e., the center line of the plasma region) and detected by the r-probe situated at the distance of $350 \mathrm{~mm}$ from the electron beam inlet. Hereafter, the position of r-probe is defined as the origin of $z$-axis $(z=0)$. The patterns of waves propagating radially are recorded with the separation distance $z$ from the exciting probe (one of $z$-probes) as a parameter, by using the interferometer system. The result is shown in Fig. 3, with the radial density profile of plasma. This figure may be considered to show the feature of propagation of the wave excited by the probe situated at the point of $z=r=0$. It is seen that the wave does heavily damp along the field ( $z$-direction) but can propagate with little damping across the field (r-direction). Apparently, it propagates obliquely to the magnetic field only in the inner region for the upper hybrid layer $\left(\omega^{2}{ }_{p}=\omega^{2}-\right.$ $\omega^{2}$ c). The wave damping along the field is very large near the cyclotron harmonics $\left(\omega=\mathrm{n} \omega_{c}\right.$ ), where the oblique propagation can not be observed. When only $\omega / \omega_{c}$ is nearly equal to a half integer, the damping is comparatively small and the oblique propagation can be observed.

If we plot the points of the same phase on each wave pattern, the wave surfaces are drawn in the r-z plane, which are shown in Fig. 4. The solid and dotted lines show the maximums and minimums of the wave patterns. The wave vector $\mathbf{k}$ must be perpendicular to the wave surface and its direction can be determined by using the delay line in the interferometer system, which is shown by arrows in the figure. In Fig. 4. (a), which corresponds to Fig. 3, the backward property is seen, with respect to both propagation components along and across the field. While, in Fig. 4 (b), the same property is seen with respect to the component across the field but the forward wave property with respect to that along the field. (Here, it is assumed that the group velocity $\partial \omega / \partial \mathbf{k}$, i. e., the energy flow of the wave is directed outward from the exciting probe.) These are very interesting characteristics which is peculiar to the Bernstein wave. In the former case, the absolute instability is expected by the electron beam injection but in the latter case, the convective one is done. In this paper, we are concerned with the latter case, that is, the forward wave property with respect to the propagation component along the field. 


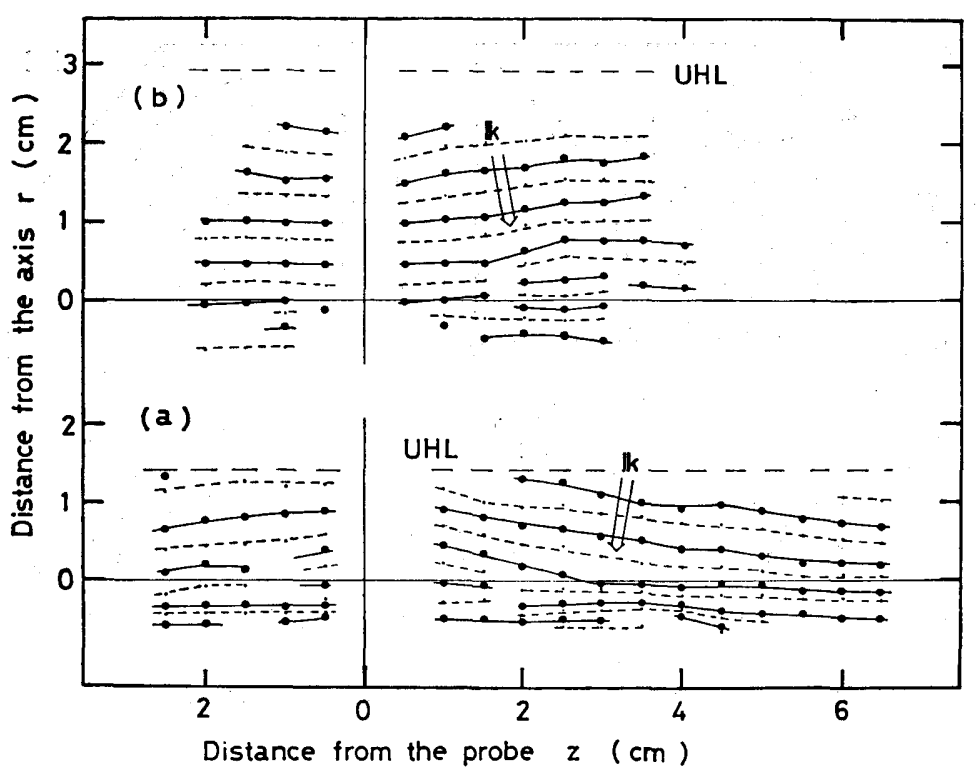

Fig. 4 The Bernstein wave surface. (a) $\omega / \omega_{c}=2.68$ and $\left(\omega_{p} / \omega_{c}\right)^{2}=9$. (b) $\omega / \omega_{c}=1.55$ and $\left(\omega_{p} / \omega_{c}\right)^{2}=6.0$. UHL shows the upper hybrid layer and arrows do the directions of the phase velocities.

\subsection{Comparison with the calculated dispersion relation}

From the experiment of wave propagation described above, the excited wave is not the standing one but propagates both radially and axially, and the diameter of plasma corresponds to about five or six wavelengths. Therefore, the plasma may be considered to be infinite and uniform for the wave, so that we can analyse the experimental results approximately by using the well-known dispersion relation for the uniform and Maxwellian plasma,

$$
\begin{aligned}
& \mathbf{K}\left(\omega, \mathbf{k}_{\|}, \mathbf{k}_{\perp}\right)=1+\frac{\omega^{2} p}{\mathbf{k}^{2} \mathbf{v}^{2} t}[ 1+\sum_{\mathrm{n}=-\infty}^{\infty} \exp (-\lambda) \mathbf{I}_{n}(\lambda) \\
&\left.\frac{\omega}{\sqrt{2} \mathbf{k}_{\|} \mathbf{v}_{t}} \mathrm{Z}\left(\frac{\omega-\mathrm{n} \omega_{c}}{\sqrt{2} \mathbf{k}_{\|} \mathbf{v}_{t}}\right)\right]=0,
\end{aligned}
$$

where $\mathrm{v}_{t}$ is the thermal velocity of plasma electron, $\lambda=\left(\mathrm{k}_{\perp} \mathrm{v}_{t} / \omega_{c}\right)^{2}, \mathrm{I}_{n}$ is the Bessel function of second kind and $Z$ is the plasma dispersion function defined by Fried and Conte. ${ }^{6}$. Under a typical plasma parameter $\left(\omega_{p} / \omega_{c}\right)$, eq. (1) is calculated for real wave vector $\mathbf{k}$ and the complex frequency $\omega+\mathbf{i} \omega_{i}$, which is shown in Fig. 5. From Fig. 5 (a), it is known that both forward $\left(\partial \omega / \partial k_{\|} \cdot k_{\|}>0\right)$ and backward $\left(\partial \omega / \partial k_{\|} \cdot k_{\|}\right.$ $<0)$ waves with respect to the propagation component along the field can appear, which explains the experimental results qualitatively. Moreover, it is seen in Fig. 5 (b), that the damping rate $\left(\omega_{i}\right)$ increases with $k_{\|}$increased and the increase is larger near the electron cyclotron harmonics because of the cyclotron damping, which 

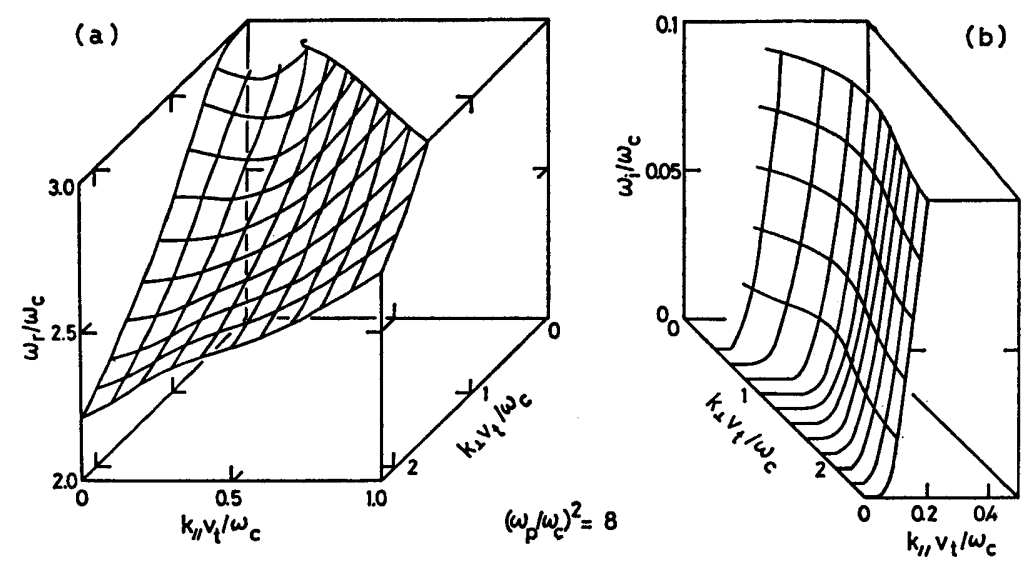

Fig. 5 The dispersion relation surface calculated using eq. (1).

$\begin{array}{lll}\text { (a) the frequency } \omega \text { v. } s \text {. wave vectoe } k & \text { (b) the }\end{array}$

damping rate $\omega_{i} \mathrm{v}$. s. wave vectork $\quad\left(\omega_{p} / \omega_{c}\right)^{2}=8$.

is consistent with the intuitive consideration. The wave can not exist for the large value of $k_{\|}$because of the large damping rate, while it can propagate with little damping, when $\omega / \omega_{c}$ is nearly equal to half integer and $\mathbf{k}_{\|} \mathbf{v}_{t} / \omega_{c}$ is much smaller than unit, i. e., the condition $\left|\omega / \omega_{c}-n\right| \gg k_{\|} v_{t} / \omega_{c}$ is satisfied for all integer $n$. As our experimental condition satisfies the above one, the oblique propagation can be observed.

Under this condition, eq. (1) is rewritten approximately as follows,

$$
\begin{aligned}
\mathrm{K}\left(\omega, \mathbf{k}_{\|}, \mathrm{k}_{1}\right)=1-\frac{\omega^{2}{ }_{p}}{\omega^{2}{ }_{c}}\left[\frac{\mathbf{k}^{2} \perp}{\mathbf{k}^{2}} \sum_{n=1}^{\infty} \frac{\exp (-\lambda) \mathrm{I}_{0}(\lambda) \mathbf{n}^{2} \omega^{2} c}{(\lambda / 2)\left(\omega^{2}-\mathbf{n}^{2} \omega^{2} \mathrm{c}\right)}\right. \\
\left.+\frac{\mathbf{k}^{2} \|}{\mathbf{k}^{2}} \exp (-\lambda) \mathrm{I}_{0}(\lambda)\right]=0 .
\end{aligned}
$$

In Fig. 6, are shown the dispersion relation surfaces calculated using eq. (2) for the experimental conditions corresponding to Fig. 4 (a) and (b). Because experimentally determined points $(\omega, \mathbf{k})$, shown by dark circles in the figure, lies near the

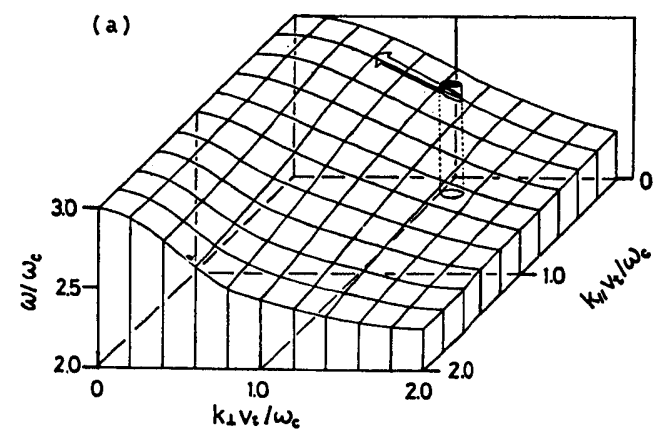

Fig. 6 The dispersion relation surface calculated using eq. (2). (a) $\omega / \omega_{c}=2$. 68 and $\left(\omega_{p} / \omega_{c}\right)^{2}=9$.

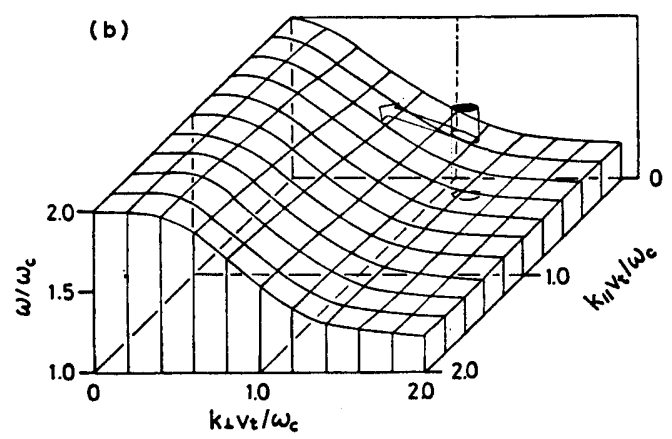

(b) $\omega / \omega_{c}=1.55$ and $\left(\omega_{p} / \omega_{c}\right)^{2}=6$. 
surface, eq. (2) is considered to be good approximation. Arrows show the directions of group velocity of the wave, i. e., the directions of $\partial \omega / \partial \mathbf{k}$. In Fig. 6 (a), it is seen that the wave shows the backward property along the field as well as across the field, while in Fig. 6 (b); the wave is forward with respect to the component along the field, which supports our exprimental results shown in Fig. 4 (a) and (b).

\subsection{Observation of the amplification of the wave due to the convective instability}

The convective instability is expected to occur for the case of forward wave along the field, while the absolute one is done for the backward wave along the field. The former is investigated here and the latter will be reported elsewhere."

Under the experimental condition where the former wave is observed, when an electron beam is injected and its density $n_{b}$ is increased at constant value of $V_{b}$, the damping factor of the wave excited by a probe decreases and then the amplification of wave is observed above the threshold value of $\mathrm{n}_{b}$, where the convective growth gets over the Landau and/or cyclotron damping. In Fig. 7 (a), the patterns of the propagating waves along the axial direction are shown with the beam current $I_{b}$ as a parameter. The direction of the phase velocity determined by the delay line is coincident with the direction of electron beam velocity. The amplification factor $k_{\| i}$, i. e., the imaginary part of $k_{\|}$, is estimated from the figure and shown in Fig. 7 (b). It is seen that when the beam is not injected and the system is in thermal equilibrium, the excited wave is heavily damped, while with the beam current increased, $\mathrm{k}_{\| i}$ also increases and becomes positive, so that for an intense electron beam the wave is amplified by the effect of the convective instability.

In Fig. 8 (a) the wave patterns along the axial direction are shown with the beam voltage $V_{b}$ as a parameter. The wave length $\lambda_{\|}$is measured from this figure and the phase velocity component $\mathrm{v}_{p \|}\left(=\omega \lambda_{\|} / 2 \pi\right)$ is estimated, which is plotted in Fig. $8(\mathrm{~b})$ as a function of $V_{b}$. The solid line in the figure shows the electron beam velocity $\mathrm{v}_{b} \|$ estimated from $\mathrm{V}_{b}$. Both

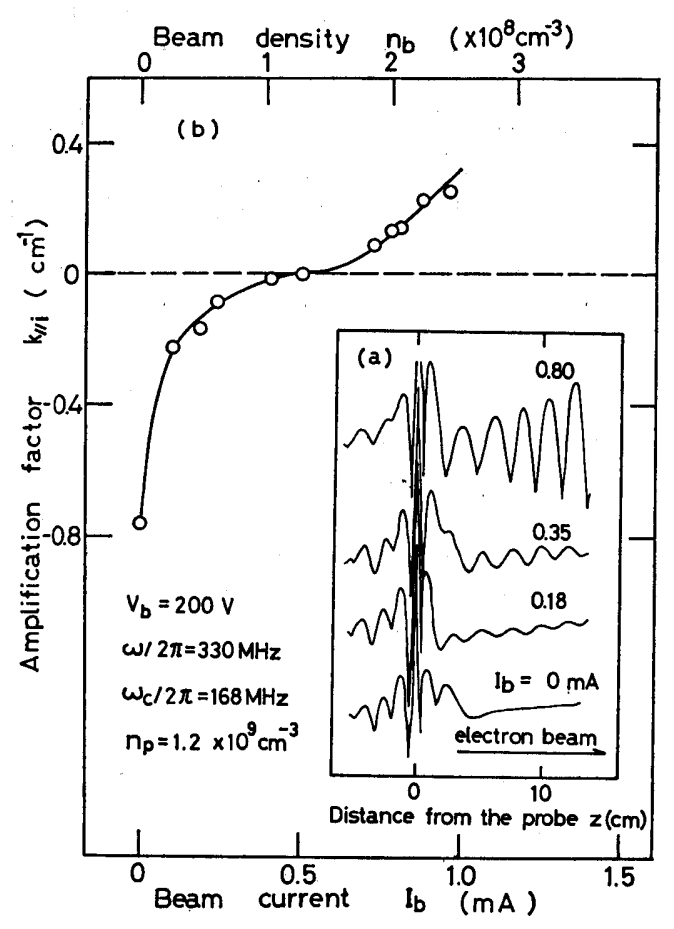

Fig. 7 (a) The wave patterns propagating along the axial direction with the beam current $\mathrm{I}_{b}$ as a parameter. $\mathrm{Ar}, \mathrm{p}=7.5 \times 10^{-4}$ torr, $\mathrm{I}_{d}=4.5 \mathrm{~mA}$ $\left(\mathrm{n}_{p}=1.5 \times 10^{\circ} \mathrm{cm}^{-8}\right)$ and $\mathrm{T}_{e}=7.8 \mathrm{eV}$. (b) The amplification factor $\mathrm{k}_{\| i}$ as a function of $\mathrm{I}_{b}$ 
the velocity $v_{p} \|$ and $v_{b} \|$ are almost equal $\left(v_{p} \approx \approx v_{b} \|\right)$, which verifies that the amplification of the wave is due to Cherenkov type excitation.

The wave patterns propagating along the radial direction are observed with the axial distance $z$ from an exciting probe as a parameter, which is shown in Fig. 9 .

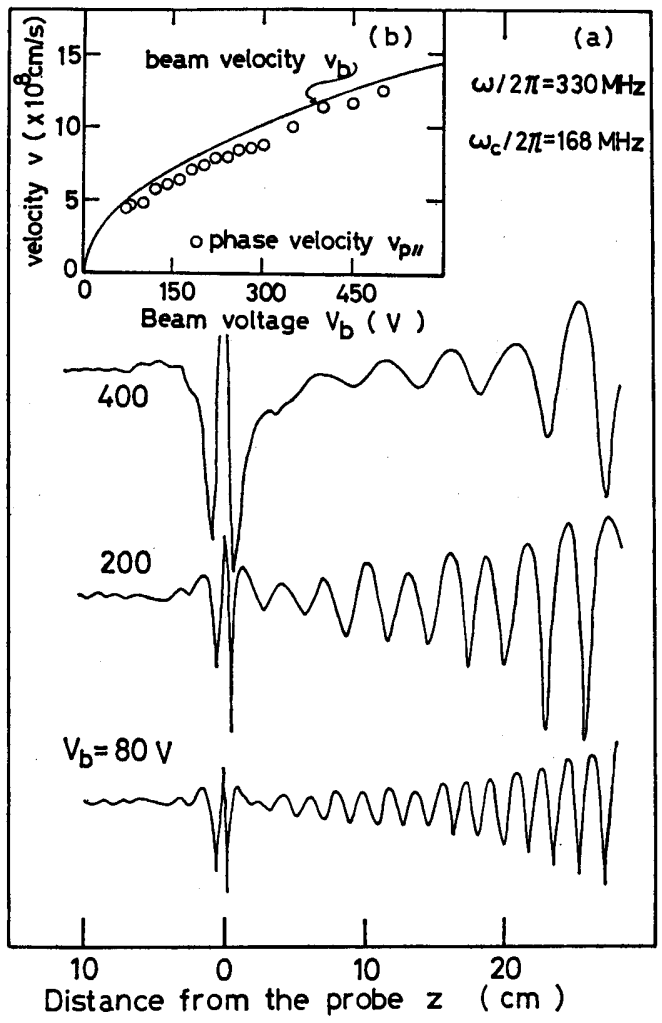

Fig. 8 (a) The wave patterns propagating along the axial direction, with the beam voltage $V_{b}$ as a parameter. Ar, $\mathrm{p}=7.5 \times 10^{-4}$ torr, $\mathrm{n}_{p}=1.2 \times 10^{9} \mathrm{~cm}^{-8}$ and $\mathrm{I}_{d}=4.6 \mathrm{~mA}$.

(b) The phase velocity component $\mathrm{v}_{p} \|(=\omega / \mathrm{k} \|)$ and the beam veloc. ity $v_{b} \|$ as functions of $v_{b}$.

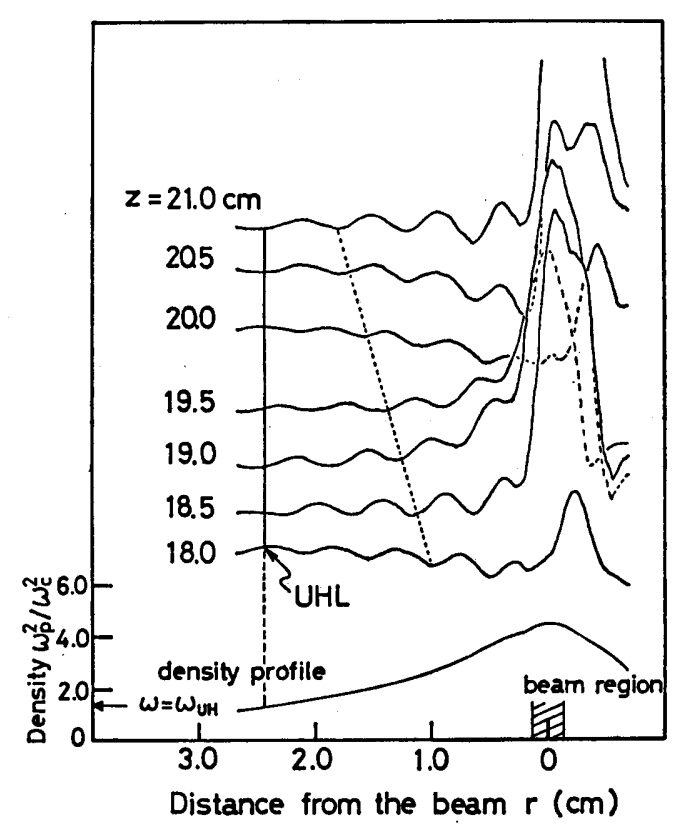

Fig. 9 The wave patterns propagating radially in a beam plasma system as the distance from the exciting antenna $z$ as a parameter and the radial density profile of plasma. A dotted line shows the same phase points of wave and a broken one does the upper hybrid layer (UHL). $V_{b}=150 \mathrm{~V},\left(\omega_{p} / \omega_{c}\right)^{2}=4.6$ and $\omega / \omega_{c}=1.55$.

Plotting the maximums and minimums of these wave patterns on the $r-z$ plane, we can obtain the surfaces of obliquely propagating Bernstein wave as shown in Fig. 10 (a), where the arrow indicates the direction of the phase velocity dertermined by using the delay line. It shows that the observed wave is confined within the upper hybrid layer, which is consistent with the works of other authors. ${ }^{8)}$ Fig. 10 (b) shows the observed wave surfaces with the directions of the phase velocities (denoted by arrows) under the different experimental condition. Considering that the energy source for amplifying the wave is localized at the beam region, the energy flow carried by the wave, i. e., the group velocity of the wave, is considered to be directed 
outward from the beam region. Therefore the wave shown in Fig. 10 (a) (where $\omega$ $\ll \omega_{u h}, \omega_{u h}$ is the upper hybrid frequency) and one of the waves in Fig. 10 (b) (where $\omega \gtrless \omega_{u h}$ ) are backward waves and the other in Fig. 10 (b) is a forward wave with respect to the radial direction. This is qualitatively consistent with the theoretical consideration described above.
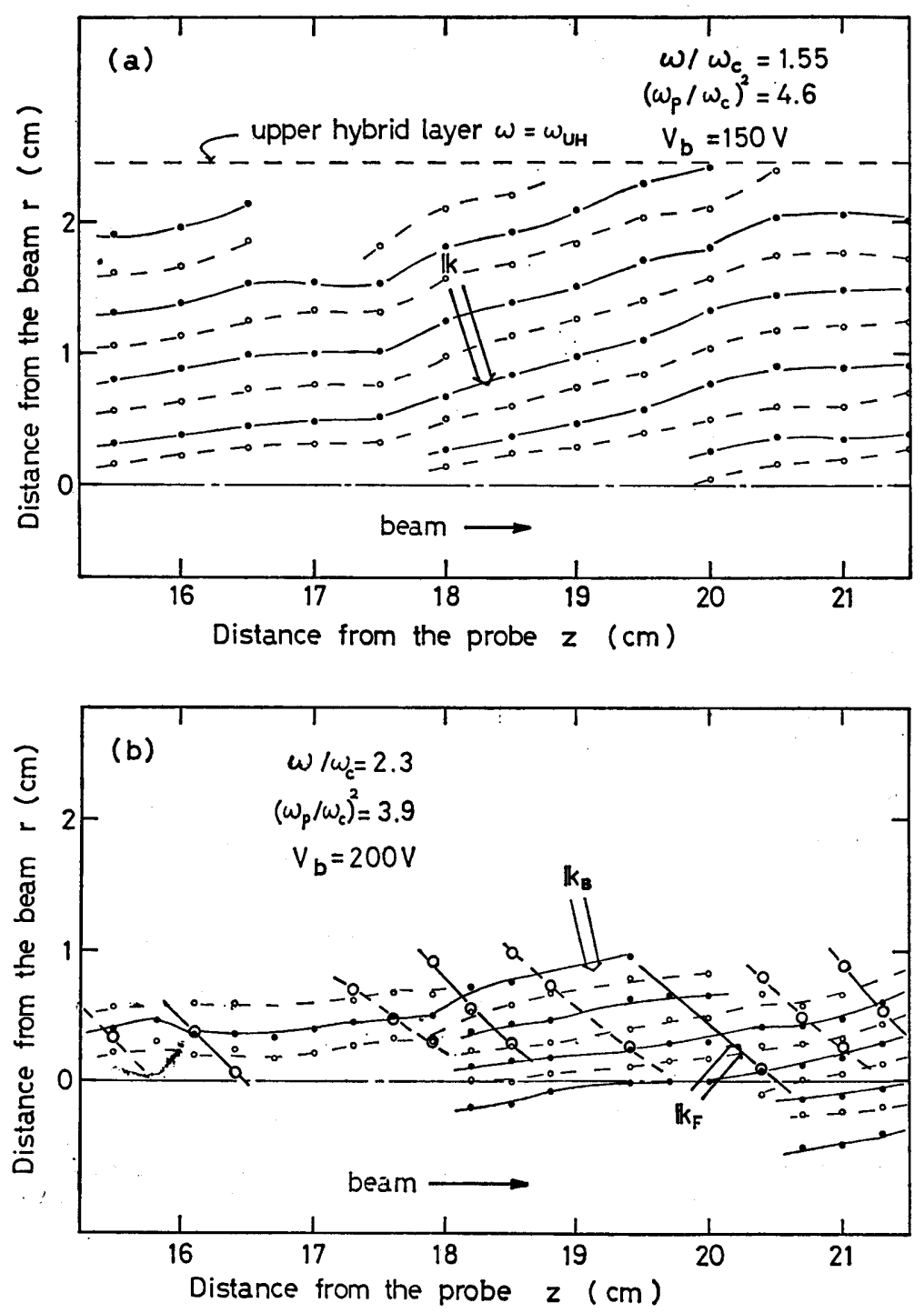

Fig. 10 The surface of the Bernstein wave propagating obliquely. The solid and dotted lines connect the points of the maximums and minimums of the wave patterns. Arrows show the directions of phase velocities $\mathrm{v}_{p}$. Ar, $\mathrm{p}=7.5 \times 10^{-4}$ torr.

(a) $\mathrm{V}_{b}=150 \mathrm{~V}, \quad \mathrm{I}_{b}=1.2 \mathrm{~mA}, \quad \mathrm{I}_{d}=8.0 \mathrm{~mA}, \omega / 2:=260 \mathrm{MHz}$ $\omega / \omega_{c}=1.55$ and $\left(\omega_{p} / \omega_{c}\right)^{2}=4.6$.

(b) $\mathrm{V}_{b}=200 \mathrm{~V}, \mathrm{I}_{b}=1.5 \mathrm{~mA}, \mathrm{I}_{d}=4.2 \mathrm{~mA}, \omega / 2 \pi=386$ $\mathrm{MHz}, \omega / \omega_{c}=2.3$ and $\left(\omega_{p} / \omega_{c}\right)^{2}=3.9$. 
In Fig. 11, the relation between the wave number component $\left(k_{\|}\right.$and $k_{\perp}$ ) of the amplifying wave which gives the angle $\theta$ of propagation $\left(\tan \theta=k_{\perp} / k_{\|}\right)$, is shown with the plasma density $\mathrm{n}_{p}\left(\propto \omega^{2}{ }_{p} / \omega^{2} c\right)$ as a parameter. For rather small density ( $\omega_{u h}$ $\lesssim \omega$ ), two values of $k_{\perp}$ correspond to each value of $k_{\|}$, the smaller one showing the forward wave and the larger one the backward wave with respect to radial direction. However, for large density $\left(\omega_{u n} \geqslant \omega\right)$, one value of $k_{\perp}$ corresponds to each value of $k_{\|}$, which shows that only the backward wave exists. The solid and broken lines show the theoretical results calculated using the dispersion relation for infinite plasma described by eq. (2) and eq. (1), respectively, which is good agreement with the experimental results. A little quantitative disagreement may result from the fact that the experimentally observed wave is a cylindrically symmetric wave in a finite system.

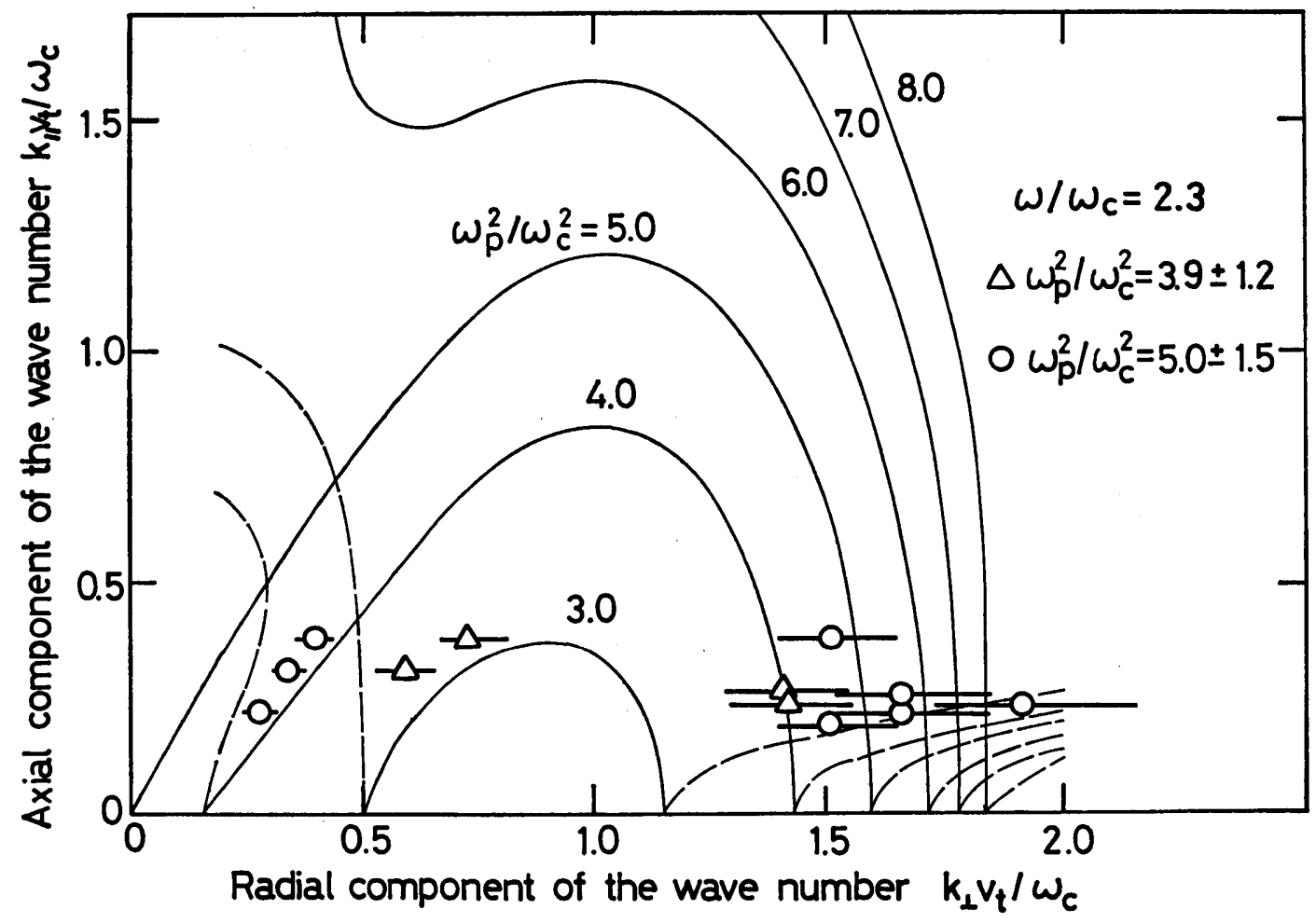

Fig. 11 The relation between the radial and axial components of the wave number. The solid and dotted curves show the calculated ones by using eq. (2) and eq. (1), respectively:

\subsection{Spontaneous excitation of the wave due to the convective instability of the thermal noise of plasma.}

When the rather weak electron beam is injected, only the externally excited wave is amplified but the spontaneous excitation of the wave is not observed, as described above. However, when a more intense electron beam is injected, the wave is excited 
spontaneously, though the external signal is not transmitted.

The curve (a) in Fig. 12 shows the distribution of the excited power along the axis, which sets on at a certain distance from the beam inlet, increases exponentially and saturates finally.

This feature is similar to other experimental results." The curve (b) in the figure shows the self-correlation of the spontaneously excited wave measured by using the r-probe situated at $z=0$ and one of $z$-probes movable axially. The direction of phase velocity determined by the delay line coincides with the direction of electron beam velocity and its value $v_{p} \|$ calculated from the wavelength $\lambda_{\|}\left(v_{p} \|\right.$ $\left.=\omega \lambda_{\|} / 2 \pi\right)$ is nearly equal to the beam velocity component $\mathrm{v}_{b \|}\left(\mathrm{v}_{p} \| \lesssim \mathrm{v}_{b \|}\right)$, therefore the excitation mechanism is Cherenkov type.

In Fig. 13, the power distribution of excited wave picked up by r-probe is shown in $n_{p}-\omega$ space with the parameter of beam keeping at constant. Solid curves show the equi-intensity ones and the value of $\mathrm{P}$ is that compared with the power of $1 \mu \mathrm{V}$ for impedance of $50 \Omega$.

On the other band, if we insert one of $z$-probes at the position denoted by ' $E P$ ' in Fig. 12 where the spontaneous excitation does not set on, and excite the wave externally, the wave is amplified by the electron beam before the spontaneous excitation does set on. Using the other of $z$-probes, the wave signals propagating along an axis are measured and the wave patterns are recorded by the interferometer system. The behavior is shown in

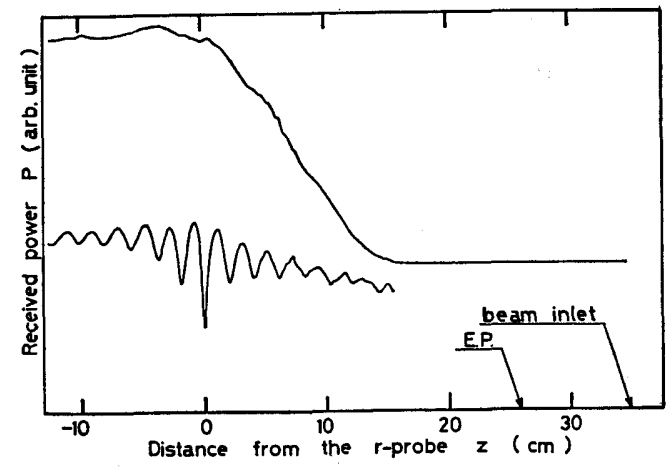

Fig.12 The power distribution of the spontaneously excited wave along the axial direction and self-correlation patterns of the wave. $\mathrm{V}_{b}=210 \mathrm{~V}, \mathrm{I}_{b}=2.75 \mathrm{~mA},\left(\omega_{p} / \omega_{c}\right)^{2}=$ 9.5 and $\omega / \omega_{c}=2.38$.

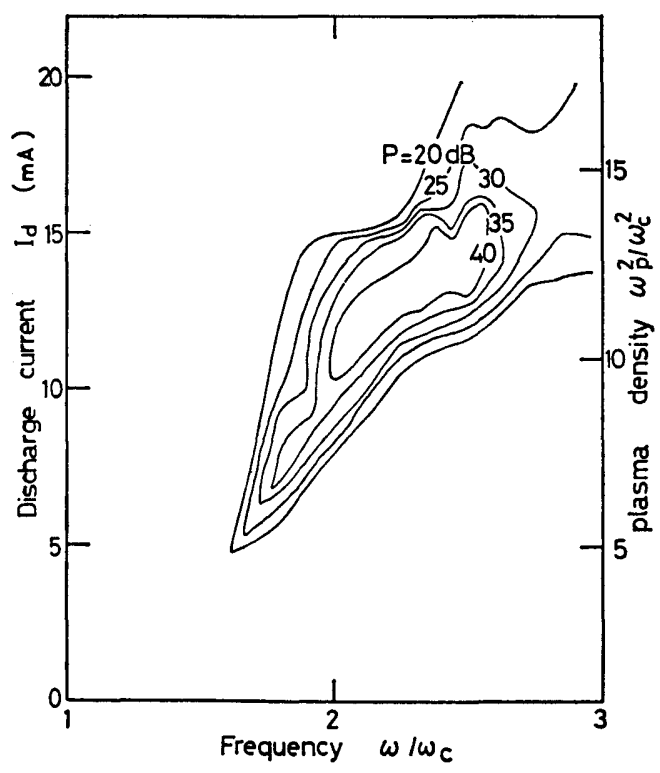

Fig.13 The power distribution of the spontaneously excited wave observed by $\mathrm{r}$-probe. $\mathrm{V}_{b}=145 \mathrm{~V}$ and $\mathrm{I}_{b}=1.5 \mathrm{~mA}$. Fig. 14 (a) with $I_{d}$ (or plasma density) as parameter at the constant frequency and the constant parameters of electron beam. It is seen that the amplification factor $k_{\| i}$ varies with $I_{d}$ but the wavelength remains at constant value which satisfies the Cherenkov excitation condition. The 
distribution of factor $k_{\| i}$ calculated from Fig. 14 (a) and many similar figures is shown in Fig. 14 (b), with the parameter of beam keeping at the same value as Fig. 13. The solid curves show the equi-amplification-factor lines. Comparing the figure with Fig. 13, the region in $n_{p}-\omega$ space where the spontaneous excitation is observed does coincide approximately with that where $\mathrm{k}_{\| i}$ is larger than $3.0 \times 10^{-2} \mathrm{~cm}^{-1}$. This fact may verify that the spontaneous excitation of the wave is the manifestation of the thermal noise amplified by the convective instability satisfying the Cherenkov excitation condition.

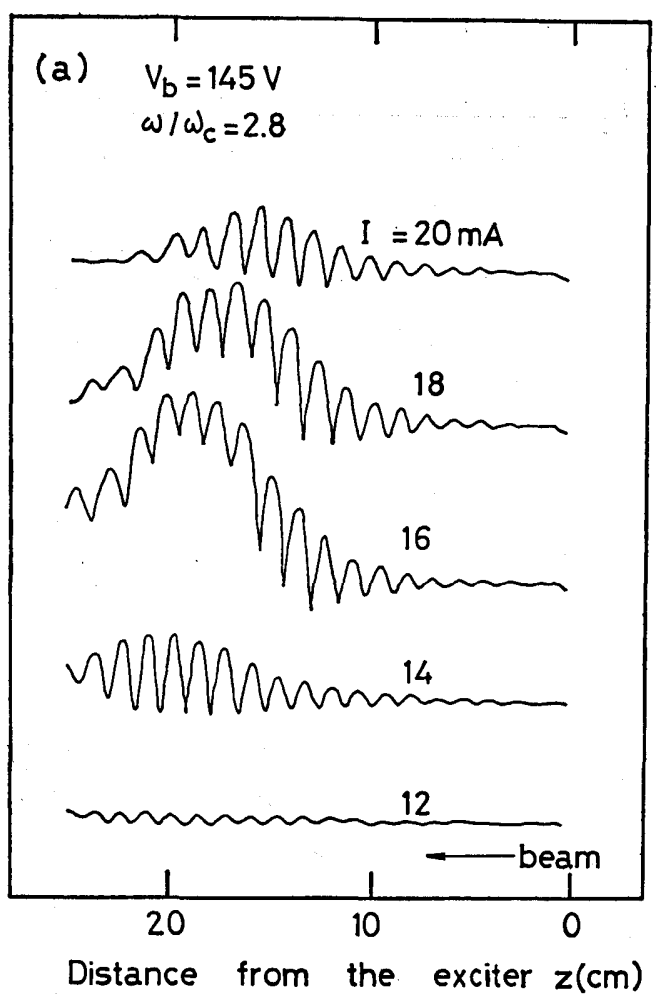

Fig.14 (a) The wave patterns propagating along the axial direction with $I_{d}$ as a parameter. $\quad V_{b}=145 \mathrm{~V}$, $I_{b}=1.5 \mathrm{~mA}$ and $\omega / \omega_{c}=2.8$.

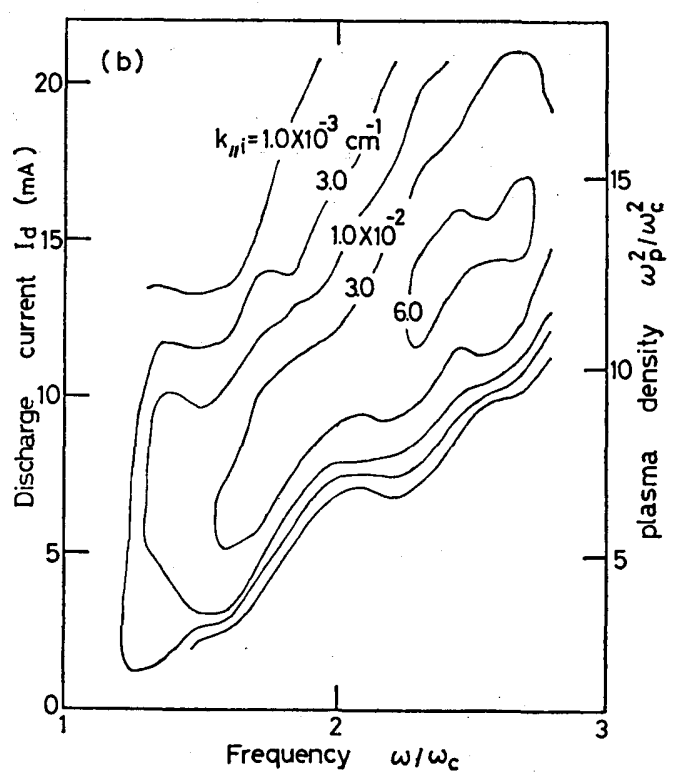

(b) The distribution of the amplification factor $k \| i$ calculated from the propagating wave patterns. $V_{b}=145 \mathrm{~V}$ and $\mathrm{I}_{b}=1.5 \mathrm{~mA}$.

\section{Conclusion}

In order to investigate the mechanism of an instability of Bernstein wave in a beamplasma system, the comparison with the study of the wave propagation must be done. The propagation of the wave in a Maxwellian plasma is studied in detail and then, the instability in a plasma penetrated by an electron beam is done for various plasma and beam parameters. The results can be described in brief as follows.

i) In a Maxwellian plasma, the Bernstein wave excited by a coaxial probe can 
propagate obliquely to the magnetic field at the frequency far from the cyclotron harmonics $\left(\left|\omega-n \omega_{c}\right| \gg k_{\|} v_{t}\right)$ but can do only perpendicularly to the field near the cyclotron harmonics because of heavy Landau and/or cyclotron damping along the field.

ii) The measurement of the propagating wave surface shows that with respect to the propagation component along the field, the forward and/or backward waves can exist, and the observed results can be explained consistently by the calculated one for an infinite and uniform plasma, because the diameter of plasma is much larger than the radial wavelength.

iii) In the case where the forward wave exists, the amplification of the externally excited wave due to the convective instability is observed when a rather weak electron beam is injected. The wave number component $k_{\|}$satisfies the Cherenkov excitation condition and the component $k_{L}$ is determined from the dispersion relation $\mathrm{K}\left(\omega, \mathrm{k}_{\|}, \mathrm{k}\right)=0$.

iv) When a more intense electron beam is injected, the spontaneous excitation of the wave is observed. From comparison of the power distribution of excited waves with the distribution of the amplification factor $\mathrm{k}_{\| i}$ in $\mathrm{n}_{p}-\omega$ space, the excited wave can be considered to be the manifestation of the thermal noise in plasma amplified by the convective instability satisfying the Cherenkov excitation condition.

When the backward wave exists, the absolute instability is expected to occur by injecting an electron beam. ${ }^{10}$ ) This instability is under study and will be reported elsewhere in the near future.

\section{Acknowledgements}

The authors wish to express their sincere thanks to Professor S. Tanaka, Kyoto University for his encouragement and valuable discussions. They also thanks to Mr. N. Miyama for his assistance on the experiment. This work was partially supported by a Grant in Aid from the Ministry of Education in Japan.

\section{References}

1) I. B. Bernstein ; Phys. Rev. 109 (1958) 10.

2) J. A. Tataronis and F. W. Crawford ; J. Plasma Phys. 4 (1970) 231, and 4 (1970) 249.

3) S. Gruber and G. Bekefi; Phys. of Fluids 11 (1968) 122.

F. Leuterer ; Plasma Phys. 11 (1969) 615.

4) M. Seidl and P. Sunka; Nucl. Fusion 7 (1967) 237.

B. R. Kusse and A. Bers; Phys. of Fluids 13 (1970) 2372.

T. Idehara, K. Ohkubo and S. Tanaka; J. Phys. Soc. Japan 27 (1969) 187.

5) K. Takayama, M. Ohtsuka, Y. Tanaka, K. Ishii and Y. Kubota; in Proceedings of the Seventh International Conference on Phenomena in Ionized Gases (Gradevinska, Knjiga, Beograde, Yugoslavia, 1966) vol. I p. 420

6) B. D. Fried and S. D. Conte; The Plasma Dispersion Function (Academic Press, New 
York, 1961).

7) T. Idehara, M. Takeda, N. Miyama and Y. Ishida; to be published.

8) S. Hiroe and H. Ikegami ; J. Phys. Soc. Japan 34 (1973) 522.

9) T. Idehara, K. Ohkubo and S. Tanaka ; J. Phys. Soc. Japan 30 (1971) 1169.

K. Mizuno and S. Tanaka; Phys Rev. Letters 29 (1972) 45, and J. Phys. Soc Japan 35 (1973) 1753. 